\title{
Beat-Based Synchronization and Steering for Groups of Fixed-Wing Flying Robots
}

\author{
Sabine Hauert, Severin Leven, Jean-Christophe Zufferey, and Dario Floreano
}

\begin{abstract}
Groups of fixed-wing robots can benefit from moving in synchrony to share sensing and communication capabilities, avoid collisions or produce visually pleasing choreographies. Synchronous motion is especially challenging when using fixed-wing robots that require continuous forward motion to fly. For such platforms, performing trajectories with forward speed lower than the minimum speed of the robot can only be achieved by acting on its heading turn rate. Synchronizing such highly dynamical systems would typically require position information and entail frequent sensing and communication among robots within the group. Instead here we propose a simple controller that reacts to regular beats received through wireless transmissions. Thanks to these beats, robot headings synchronize over time. Furthermore, these controllers can easily be parameterized to steer and regulate the global progression speed of groups of robots. Experiments are performed both in simulation and using up to five fixed-wing flying robots.
\end{abstract}

\section{Introduction}

Flying robots are often required to follow trajectories that can be easily steered and speed-regulated for real-world applications such as teleoperation, visiting areas of interest, exploration and tracking [14]. In the case of fixed-wing platforms, this means setting the turn rate of the robot such that it will perform loitering trajectories with a given global speed and direction. Indeed, unlike ground robots or rotorcrafts, fixed-wing robots need to maintain their flight velocity within a certain limit to avoid stalling. Loitering allows robots to slow down their global progression speed.

Moreover, some applications can benefit from deploying several robots rather than a single one to increase the number of sensors in the air, produce different

Sabine Hauert · Severin Leven · Jean-Christophe Zufferey · Dario Floreano

Laboratory of Intelligent Systems, Ecole Polytechnique Fédérale de Lausanne, Switzerland e-mail: \{sabine.hauert, severin.leven\} @epfl.ch \{jean-christophe.zufferey, dario.floreano\}@epfl.ch 
points of view and for increased mission robustness [14]. Synchronizing the heading of the robots within a group can further allow them to move coherently in a given direction, which can help them avoid collisions, maintain relative distance among robots for sensor fusion and favor communication [1].

Synchronizing loitering trajectories in real-time across robots while respecting commands in terms of global motion direction and speed is challenging. Work on formation path following for unicycle-type vehicles has so far concentrated on mathematical models and simulations built upon the assumption that robots know the precise relative position of neighbors (range and bearing) and sometimes their heading and speed [2, 3, 8, 10]. Using this knowledge, robots continuously align their position to that of their neighbors and to the trajectory they need to follow. However, so far no results have been demonstrated with real flying robots and simulations only depict scenarios without sensor noise or low forward speeds and limited turning rates unrealistic for flying robots. Furthermore, inferring position in a robust and dependable manner is one of the main challenges in aerial robotics [5, 6].

Instead, here we present a positionless strategy to synchronize and steer groups of robots that does not require memory, computation or high-bandwidth communication. This work is inspired from the idea of emergent synchronization studied in nature [11] and the discovery of synchronized controllers for flying robots using artificial evolution [6]. We consider robots that fly at constant speed and rely on a heading sensor and a low-level autopilot that is able to regulate turn rate with some precision [7]. Based on these assumptions, we propose a minimal controller for flying robots where synchronization emerges from interactions between each robot and rhythmic beats sent using a radio-emitter from a base station on the ground or one of the robots. Each beat is a step function composed of an "on" and "off" phase of fixed duration. Notice that in the most economical mode, only "on" and "off" signals need to be sent. Based on this beat and their heading, robots will change their turn rate to achieve adequate loitering trajectories that display two essential features seen in Fig. 1. First robots, regardless of their initial heading, converge to identical headings over time (synchronization). Second, the direction and speed of the loitering trajectories can be changed to a desired value by mathematically determining the parameters of the controller. These two features are presented in this order in the paper because the steering of the robots has as a prerequisite their synchronization. Finally, results in this paper are shown both in simulation and with up to five physical fixed-wing flying platforms that are fully autonomous.

\section{Heading Synchronization}

Synchronization is essential to make robots move coherently in groups. To achieve this we developed a controller that reacts to beat signals and heading information. In particular we define a simple controller where robots receiving the "on" phase of a beat of duration $t_{1}$ perform a fixed turn rate of $\omega_{1}$. The "off" phase is then initiated for a duration $t_{2}$. During this phase, robots perform a turn rate of $\omega_{1}$ or $\omega_{2}$ depending 
Fig. 1 Example of synchronized steering. Robots launched from $(0,0)$ in opposite directions receive a beat composed of an "on" phase of duration $t_{1}$ (grey) and an "off" phase of duration $t_{2}$ (black). Over time, the robot headings synchronize. This can be seen by the fact that at each start of a beat, the headings of the two robots are identical. Furthermore, the trajectories converge to a fixed global velocity (speed and direction).

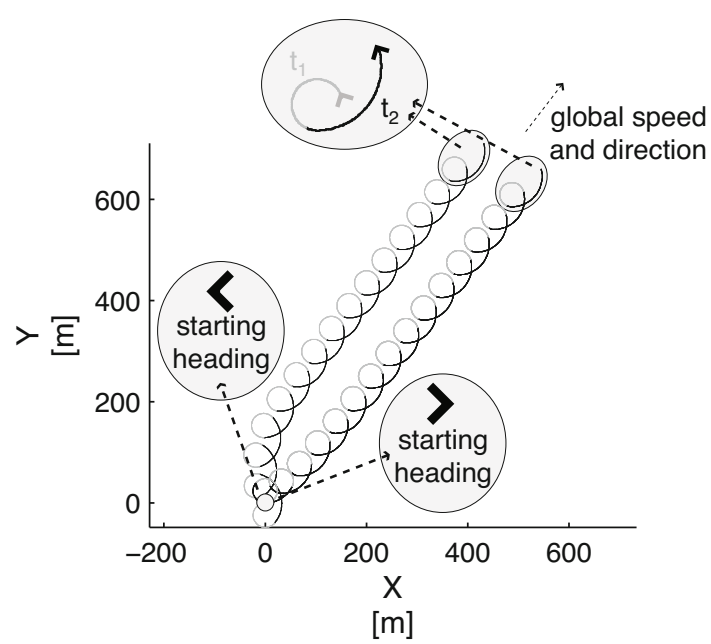

on whether the angle $\widehat{h_{\text {lim }} h}$ between a predefined heading limit $h_{\text {lim }}$ and their current heading $h$ is positive or negative. $\omega_{1}$ and $\omega_{2}$ are assumed to be of same sign. The resulting controller is described below and a possible trajectory is shown in Fig. 2

$$
\omega= \begin{cases}\omega_{1}, & \text { if beat on } \\ \omega_{1}, & \text { if beat off and } \widehat{h_{\text {lim }} h}<0 \\ \omega_{2}, & \text { if beat off and } \widehat{h_{\text {lim }} h}>0\end{cases}
$$

This controller has the property of converging to identical headings at the beginning of each beat. This can be explained by the fact that the amount of time $t_{\text {lim }}$ spent between the moment the beat is turned off and the robot reaches the heading limit $h_{\text {lim }}$ depends on the initial heading of the robot. If the robot starts at a heading as shown in Fig. 3(left), it will perform more than $2 \pi$ within one beat $\left(t_{1}+t_{2}\right)$, thereby changing its starting heading for the next beat. However, if the robot starts at the

Fig. 2 Example of a robot trajectory implementing the controller described in equation 1 Here a robot receives a beat composed of an "on" phase of duration $t_{1}$ (grey) and an "off" phase of duration $t_{2}$ (black). Using this, the robot controller sets the turn rate to $\omega_{1}$ or $\omega_{2}$ depending on the beat and the heading of the robot with respect to a predefined heading $h_{\text {lim }}$ (see dashed lines).

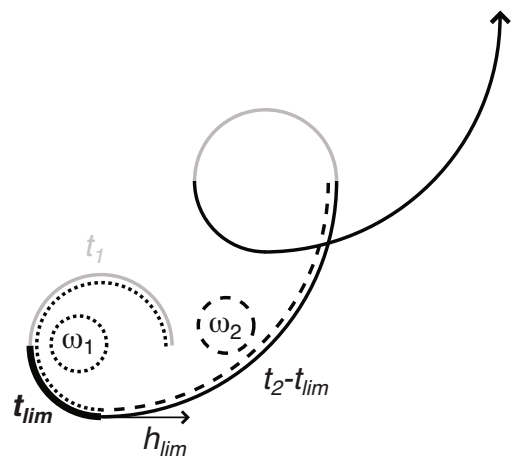




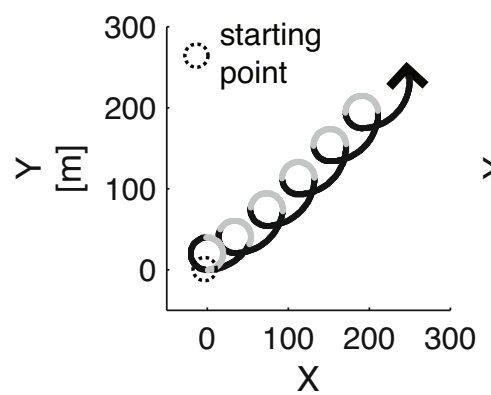

$[\mathrm{m}]$

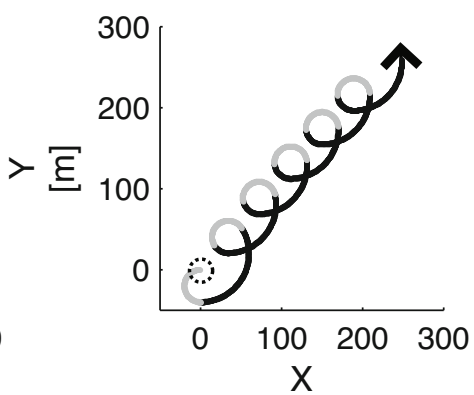

$[\mathrm{m}]$

Fig. 3 Robot trajectories synchronize over time by converging to a state where at the beginning of each beat of duration $t_{1}$ (grey) $+t_{2}$ (black), the robot returns to the same heading. Synchronization is achieve independently of the initial heading of the robot as shown in these two examples with opposite initial headings. Notice that at the end of the trajectory, robot headings are identical.

heading shown in Fig. 3](right), it will perform less than $2 \pi$ within one beat. Instead, once synchronized, the robot will perform $2 \pi$ during one beat, meaning that it will start the next beat with the same heading.

The overall effect is that robots listening to identical beats and using the same controller parameters will synchronize over time. In the particular case shown in Fig. 4] robots synchronize after 2 beats.

Assuming $\omega_{1}>\omega_{2}$, suitable parameters $\left(t_{1}, t_{2}, \omega_{1}\right.$ and $\left.\omega_{2}\right)$ that lead to trajectories that perform $2 \pi$ during one beat must be such that the minimum value for $t_{\text {lim }}$ named $t_{\text {lim_min }}$ produces trajectories that perform less than $2 \pi$ during one beat while the maximum value $t_{\text {lim } \_ \text {max }}$ produces trajectories that perform more than a full revolution during one beat. These conditions can be mathematically described as:

$$
\begin{aligned}
& \left|\omega_{1}\right| \cdot t_{1}+\left|\omega_{1}\right| \cdot t_{\text {lim_min }}+\left|\omega_{2}\right| \cdot\left(t_{2}-t_{\text {lim_min }}\right)<2 \pi \\
& \left|\omega_{1}\right| \cdot t_{1}+\left|\omega_{1}\right| \cdot t_{\text {lim_max }}+\left|\omega_{2}\right| \cdot\left(t_{2}-t_{\text {lim_max }}\right)>2 \pi
\end{aligned}
$$

where

$$
\begin{aligned}
& t_{\text {lim_min }}=t_{2}-\min \left(t_{2}, \frac{\pi}{\left|\omega_{2}\right|}\right) \\
& t_{\text {lim_max }}=\min \left(t_{2}, \frac{\pi}{\left|\omega_{1}\right|}\right)
\end{aligned}
$$

Notice that setting $\omega_{2}>\omega_{1}$ would simply result in reverting the inequalities.

As an advantage, this controller is able to compensate for perturbations and resynchronize. This is shown in Fig. 5 where we introduce 8 large perturbations to the system by increasing or decreasing the turn rate $\omega_{1}$ and $\omega_{2}$ by $0.05 \mathrm{rad} / \mathrm{s}$ and $0.1 \mathrm{rad} / \mathrm{s}$ during an entire beat. 


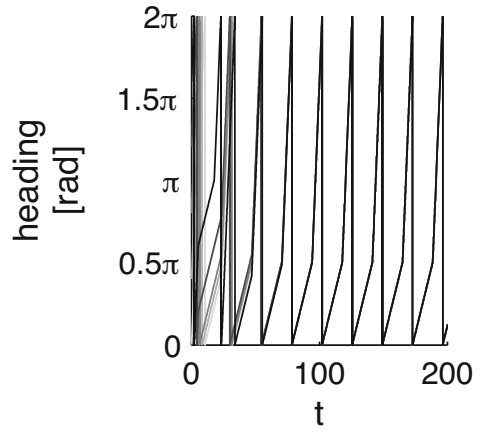

[s]

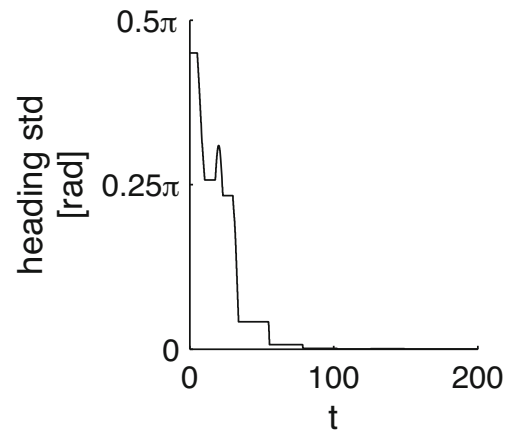

[S]

Fig. 4 Headings (left) of 5 simulated robots initialized at headings $0, \frac{2 \pi}{5}, \frac{4 \pi}{5}, \frac{6 \pi}{5}, \frac{8 \pi}{5}$. Notice that over time, the standard deviation (right) across robot headings goes down to 0 , meaning the robot are synchronized.

Fig. 5 Capacity of the robot controller to synchronize after 8 large perturbations to its turn rate

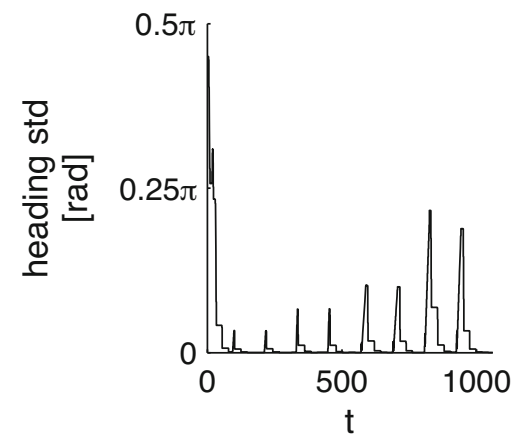

[s]

\section{Global Motion}

For real-world applications, such as target tracking or exploration, robots will be required to change their global direction and speed. Here we derive equations to identify how parameters can act on the direction $\alpha$ and global speed $v_{\text {avg }}$ of robots implementing the controller presented in Eq. 1. In particular, using symbols in Fig. 6 and knowing that robots with forward speed $v$ will perform $2 \pi$ during one beat, we can calculate

$$
\alpha=h_{\text {lim }}+\tan ^{-1} \frac{a}{b}-\beta+\frac{3 \pi}{2}
$$

and

$$
v_{a v g}=\frac{\sqrt{a^{2}+b^{2}}}{t_{1}+t_{2}}
$$


where

$$
\begin{aligned}
a & =\frac{v}{\omega_{1}} \cdot \sin (\beta)+\frac{v}{\omega_{2}} \cdot \sin (\gamma) \\
b & =\frac{v}{\omega_{2}}-\frac{v}{\omega_{2}} \cos (\gamma)-\left(\frac{v}{\omega_{1}}-\frac{v}{\omega_{1}} \cos (\beta)\right) \\
\beta & =\omega_{1} \cdot\left(t_{1}+t_{\text {lim }}\right) \\
\gamma & =\omega_{2} \cdot\left(t_{2}-t_{\text {lim }}\right) \\
t_{\text {lim }} & =\frac{2 \pi-\left|\omega_{1}\right| \cdot t_{1}-\left|\omega_{2}\right| \cdot t_{2}}{\left|\omega_{1}\right|-\left|\omega_{2}\right|}
\end{aligned}
$$

Fig. 6 Symbols used to determine the average advancement speed and direction of the robot trajectories.

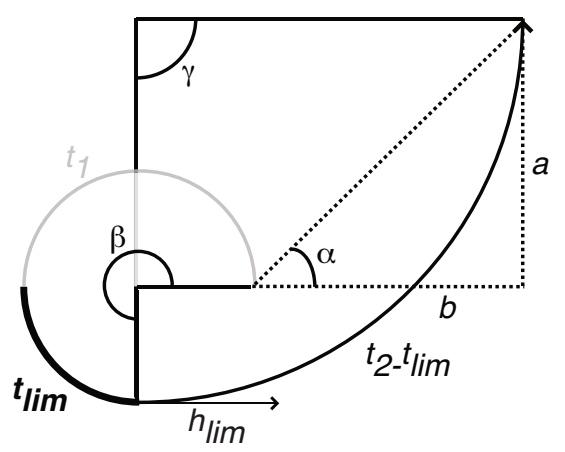

While these equations allow to predict in what direction and at what speed each robot will move, it is challenging to set the parameters in order to achieve a desired command because the parameters $\left(\omega_{1}, \omega_{2}, t_{1}\right.$ and $\left.t_{2}\right)$ can not be isolated analytically. The problem can however be simplified by only considering trajectories where

$$
\begin{aligned}
& \beta=\frac{3}{2} \pi \\
& \gamma=\frac{\pi}{2}
\end{aligned}
$$

which can be achieved if

$$
\begin{aligned}
& t_{1}=\frac{\pi}{\left|\omega_{1}\right|} \\
& t_{2}=\frac{\pi}{2\left|\omega_{1}\right|}+\frac{\pi}{2\left|\omega_{2}\right|}
\end{aligned}
$$

leading to trajectories where

$$
\begin{aligned}
\alpha & =h_{\text {lim }}+\frac{\pi}{4} \\
v_{\text {avg }} & =\frac{\sqrt{2}\left(\frac{v}{\left|\omega_{2}\right|}-\frac{v}{\left|\omega_{1}\right|}\right)}{t_{1}+t_{2}}
\end{aligned}
$$


Thanks to Eq. 17 and 18 , the parameters can easily be modified to modulate the global motion direction and speed of robot trajectories. In particular, the direction can be changed by modifying $h_{\text {lim }}$. Furthermore, increasing or decreasing the global speed of each robot can be done by increasing or decreasing the difference between $\omega_{2}$ and $\omega_{1}$ respectively within the boundaries set by Eq. 2 For negative turn rates we can use $\beta=-\frac{3}{2} \pi, \gamma=-\frac{\pi}{2}$ and $\alpha=h_{\text {lim }}+\pi-\frac{\pi}{4}$.

\section{Experimental Setup}

For the purpose of this experiment, we use up to five fixed-wing platforms that are light-weight $(420 \mathrm{~g}, 80 \mathrm{~cm})$ and safe (Fig. 7). Each robot is equipped with an autopilot for the control of altitude, airspeed and turn rate that provides an interface for receiving commands from a navigation controller. Embedded in the autopilot is a micro-controller that runs a minimalist control strategy based on input from only 3 sensors: one gyroscope and two pressure sensors [7].

Fig. 7 Safe flying wing $(450 \mathrm{~g}, 80 \mathrm{~cm})$ for outdoor experiments made out of soft material and with a back-mounted propeller. The robot is equipped with an autopilot, embedded Linux, WiFi dongle and GPS (only for logging purposes).

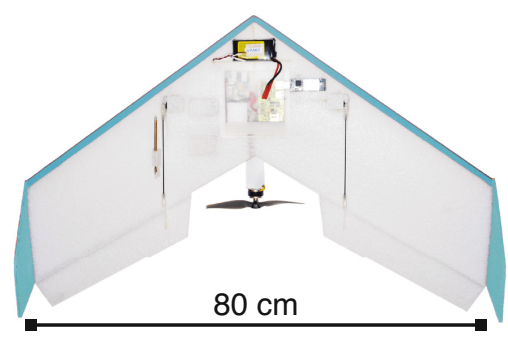

The controller presented in this paper is implemented on a Toradex Colibri PXA270 CPU board running Linux, connected to an off-the-shelf USB WiFi dongle. The output of this high-level computer, namely a desired turn rate, is sent as control command to the autopilot. Altitude is set to a different constant value for each robot between $50 \mathrm{~m}$ and $90 \mathrm{~m}$ and separated by $10 \mathrm{~m}$. The airspeed is also constant at $12 \mathrm{~m} / \mathrm{s}$. In order to log flight trajectories, the robot is further equipped with a u-blox 1 LEA-5H GPS module.

For emitting and receiving the beat, robots use a Netgeat 2 WNDA3100 dongle implementing the $802.11 \mathrm{n}$ standard and transmitting in the $5 \mathrm{GHz}$ band. This is interesting with respect to transmissions in the $2.4 \mathrm{GHz}$ band because it allows for less interference with the considerable number of devices currently used in this band. Dongles are configured for ad-hoc mode and have a communication range of nearly $500 \mathrm{~m}$ line-of-sight.

\footnotetext{
${ }^{1}$ http://www.u-blox.com

${ }^{2} \mathrm{http} / / /$ netgear.com
} 


\section{Results}

To validate the synchronization and steering of groups of robots we perform a set of in-flight experiments with up to five physical fixed-wing robots described in section 4 During these experiments, one of the robots sends beats by emitting heartbeat messages at an interval of $5 \mathrm{~ms}$ during the "on" phase and no messages during the "off" phase. This was done to increase the robustness of beat signals to communication failure.
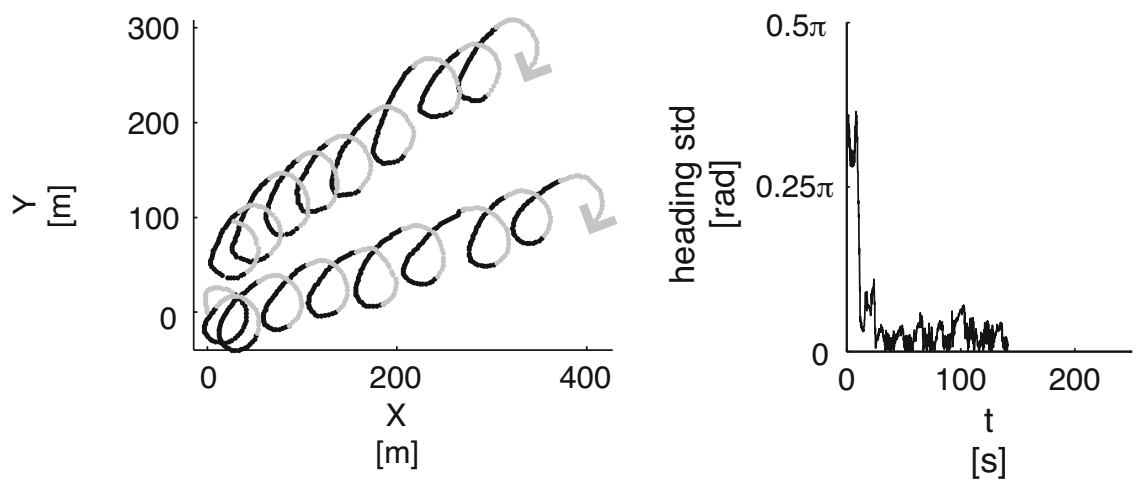

Fig. 8 Demonstration of synchronization on board two real flying robots in an outdoor experiment. Left: trajectories of the robots. Right: standard deviation on the robot headings.

The first experiment shown in Fig. 10 is aimed at demonstrating that two robots that start with different initial headings will synchronize over time. Parameters for this experiment are based on Eq. 1 with $h_{\text {lim }}=5.4 \mathrm{rad} \omega_{1}=-0.7 \mathrm{rad} / \mathrm{s}$ and $\omega_{2}=$ $-0.1 \mathrm{rad} / \mathrm{s}$ with $t_{1}$ and $t_{2}$ set following Eq. 15 and 16 respectively. Notice how the standard deviation of robot headings rapidly goes down to nearly zero, thereby indicating synchronization.

Beyond synchronization, we aim at showing that a group of two robots can be steered and speed regulated. In particular, we propose three mission goals. In the first, robots are directed to go towards the North. $h_{\text {lim }}$ is then changed, thereafter directing the group to the South (phase II). In the third phase the turn rate $\omega_{2}$ is changed to slow down the global progression speed of the group. As a result, Fig. 9 shows how the speed and direction of the robots can be changed while remaining synchronized. Parameters for this experiment are given in Table 11. Notice that because of wind to the South of around $3.5 \mathrm{~m} / \mathrm{s}$, the desired speed and direction of the group in not exact with respect to theoretical calculations. Good synchronization and group steering is however achieved.

Finally, in Fig. 10 we show that this method scales to five flying robots. For this experiment, we propose two mission goals. In the first, robots are directed to go 
Table 1 Controller parameters used to achieve trajectories shown in Fig. 9

\begin{tabular}{|c|c|c|c|}
\hline & $h_{\text {lim }}[\mathrm{rad}]$ & $\omega_{1}[\mathrm{rad} / \mathrm{s}]$ & $\omega_{2}[\mathrm{rad} / \mathrm{s}]$ \\
\hline phase I & 5.8 & -0.7 & -0.1 \\
\hline phase II & 2.7 & -0.7 & -0.1 \\
\hline phase III & 2.7 & -0.7 & -0.3 \\
\hline
\end{tabular}

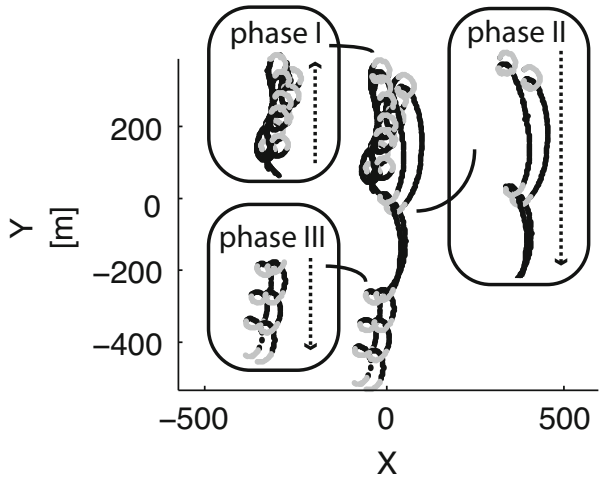

$[\mathrm{m}]$

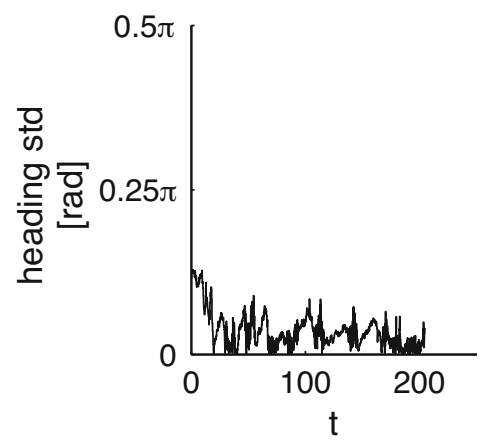

[s]

Fig. 9 Demonstration of synchronization and steering of two real flying robots in an outdoor experiment. Left: trajectories of the robots. Right: standard deviation on the robot headings. Three phases are shown here, in the phase I, the robot group is directed to the North against the wind. In phase II, robots are directed to turn around and proceed South. Phase III then shows how the robots can be slowed down. Notice that the robots remain synchronized throughout the experiment.

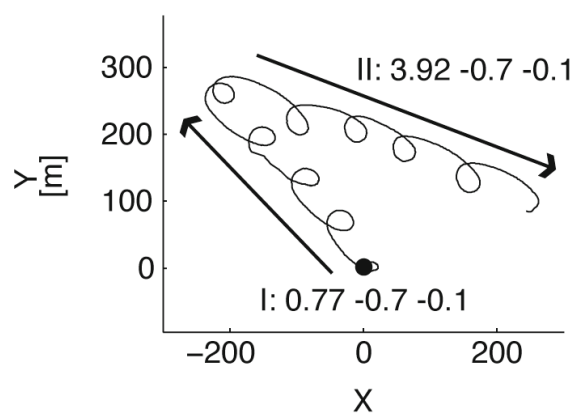

[m]

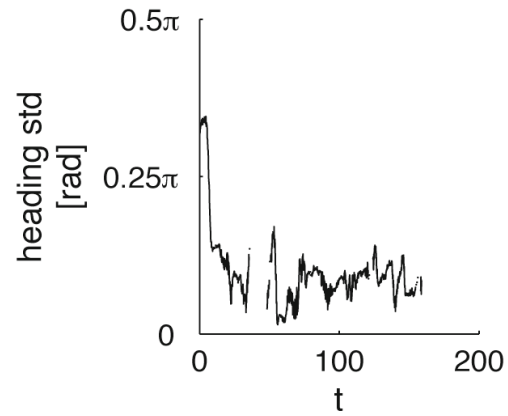

[s]

Fig. 10 Demonstration of synchronization and steering of five real flying robots in an outdoor experiment. Left: mean trajectory of the robots. Right: standard deviation on the robot headings. Two phases are shown here, in phase I, the robot swarm is directed to the West. In phase II, robots are directed to turn around and proceed East. Parameters represent $h_{\text {lim }}, \omega_{1}$ and $\omega_{2}$. Notice that the robots remain synchronized throughout the experiment which is why it is possible to plot such a mean trajectory. 
towards the West. $h_{\text {lim }}$ is then changed, thereafter directing the swarm to the East (phase II). Fig.10 (right) shows the standard deviation on the heading of the robots which rapidly decreases over time as robots synchronize. The five robot trajectories are summarized by their mean which highly resembles the individual trajectories because all robots are synchronized. In this experiment wind of around $1 \mathrm{~m} / \mathrm{s}$ to the North-East was present. Overall, robots are able to achieve good synchronization and steering. Because of wind and the dynamics of the robots, which prevent them from rapidly changing their turn rate, the actual direction performed by the swarm is slightly shifted with respect to the initial goal. A video showing the synchronization of five robots can be seen on our project webpage (http://lis.epfl.ch/smavs).

\section{Discussion}

While group steering with synchronization is a first step towards deploying flying robots in real-world applications, two main challenges persist, namely the difference in flight dynamics across platforms and the question of what application-oriented outer-loop control could be used to determine where to steer the robots.

Indeed, two robots implementing identical turn rate commands and speed commands will generally not perform identical trajectories due to sensor noise and hardware differences. The effect of turn rate bias on the synchronization and steering of the robots can be seen in Fig. 11, where we show the simulated trajectories resulting from turn rates of value $\omega_{1}=[0.6,0.65,0.7] \mathrm{rad} / \mathrm{s}$ and $\omega_{2}=0.1$ for $t_{1}=4.488 \mathrm{~s}$ and $t_{2}=17.9520 \mathrm{~s}$. However, robots that display different turn rates will still perform one revolution during one beat if they meet requirements described in Eq. 2 and 3 . Therefore, the shift in heading among the robots is stable over time.

To achieve robot behaviors as similar as possible, some feedback would be required to calibrate the robot turn rates over time. This feedback could be based on comparing the robot headings at each beat with the desired heading and modifying the turn rate accordingly.

Finally, in scenarios with wind and without any position information, it becomes challenging to know where to steer the group of robots. For this purpose, one can implement an outer-loop responsible for issuing commands for the steering and speed regulation of the robots. This outer-loop can reactively increase or decrease the speed of the swarm and make it turn more or less based on sensory input from the robots. Reactive controllers that do not use position have been developed in the past to allow flying robots to remain leashed or track a base station on the ground [4] or avoid obstacles [13]. As an example, we consider in simulation a scenario where the swarm must remain within the communication range of a base station on the ground. Each time a robot loses its connection to the base station, it records its heading and broadcasts a new set of controller parameters to all robots that make them pursue a global direction opposite from its disconnection heading. In that manner it becomes the "leader" of the swarm. Results in Fig. 12 show that robots starting from different 

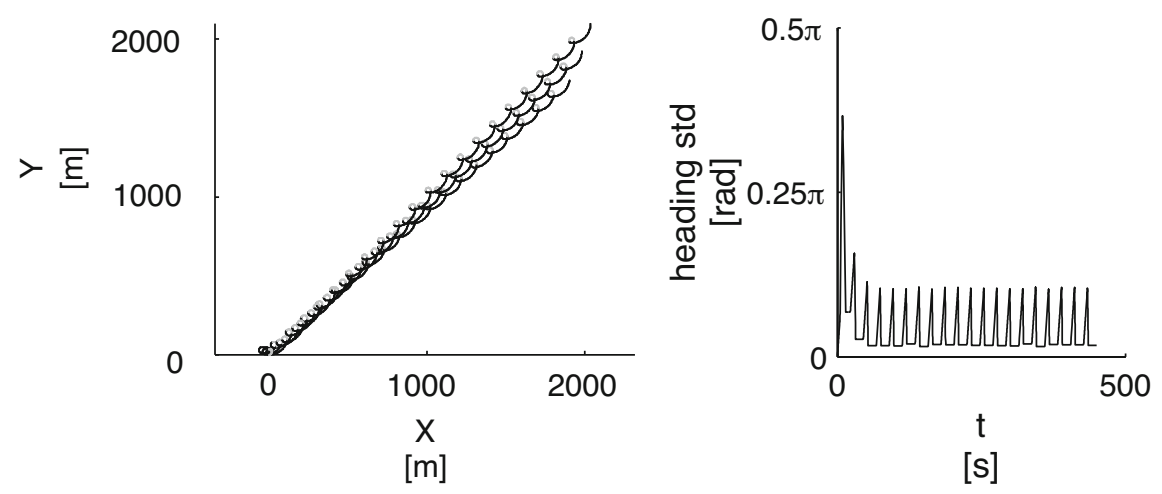

Fig. 11 Effect of turn rate bias in simulation on the robot trajectories (left) and heading (right) of robots with turn rates equal to $\omega_{1}=[0.6,0.65,0.7] \mathrm{rad} / \mathrm{s}$ and $\omega_{2}=0.1$ for $t_{1}=4.488 \mathrm{~s}$ and $t_{2}=17.9520 \mathrm{~s}$. Notice that while the robots implement different controllers, their headings still synchronize with a constant shift. The direction and advancement speed of the group is also slightly modified across robots.
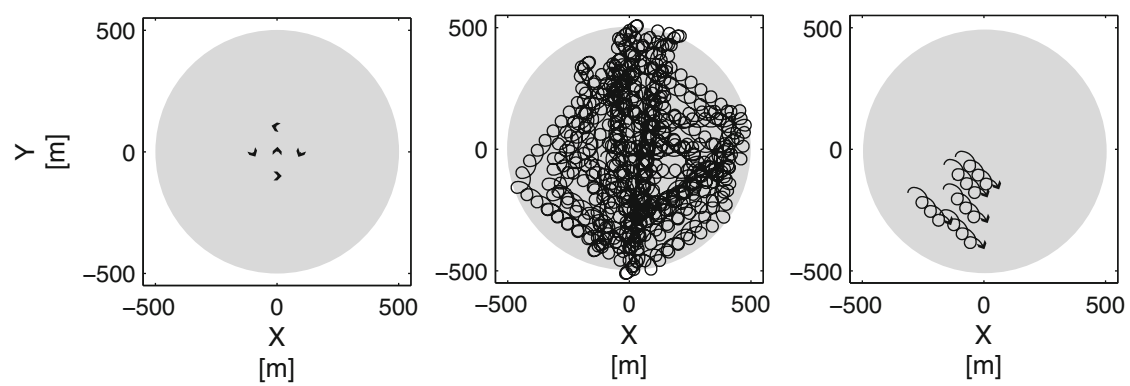

Fig. 12 Simulated swarms synchronize and move in groups while remaining connected to a base station on the ground. The grey area represents the communication range of the base station in $(0,0)$. The figure to the left shows the starting position and heading of the robots, the center image shows all trajectories over a $30 \mathrm{~min}$ trial and the figure to the right shows the end of the trial and the synchronized heading of the robots.

headings are able to synchronize and move in groups while remaining connected to the base station.

\section{Conclusion}

Steering groups of robots while keeping them synchronized is an essential building block for the deployment of flying robots in real-world applications. However, challenges arise when controlling robots with tightly constrained motion dynamics, such 
as fixed-wing platforms whose speed must remain within a certain limit. Rather than relying on complex controllers based on position, frequent sensing and communication, or memory, we proposed a minimal controller that modulates the turn rate of robots based on their current heading and a beat signal sent from a radio emitter on a ground station or a robot. This controller has the property of synchronizing the robot headings to the beats, regardless of their starting heading. Furthermore, it can easily be parametrized to steer a group of robots and change its global speed. Finally, we demonstrated synchronization and steering in reality using up to five fully autonomous fixed-wing flying robots.

To improve results in reality, two directions were suggested. The first consists in using feedback on heading to improve the accuracy of turn rate commands across robots. The second includes applying such mechanisms to real-world applications by adding an outer-loop responsible for emitting the higher-level steering commands. In the future, efforts should also be made to describe controllers in terms of synchronized oscillators [9, 12]. Such an endeavor would allow for stability proofs, more formal mathematical models and a large range of extensions based on different forms of synchronization states found in the literature.

Acknowledgements. This work is supported by armasuisse, competence sector Science + Technology for the Swiss Federal Department of Defense, Civil Protection and Sports. Sincere thanks to Steffen Wischmann for contributing his expertise in dynamical systems.

\section{References}

1. De Nardi, R., Holland, O., Woods, J., Clark, A.: SwarMAV: A swarm of miniature aerial vehicles. In: Proceedings of the 21st International UAV Systems Conference (2006)

2. Ghabcheloo, R., Pascoal, A., Silvestre, C., Kaminer, I.: Non-linear co-ordinated path following control of multiple wheeled robots with bidirectional communication constraints. International Journal of Adaptive Control and Signal Processing 21(2-3), 133157 (2007)

3. Ghommam, J., Saad, M., Mnif, F.: Formation path following control of unicycle-type mobile robots. In: IEEE International Conference on Robotics and Automation, pp. 1966-1972. IEEE Press, Piscataway (2008)

4. Hauert, S., Leven, S., Zufferey, J.C., Floreano, D.: Communication-based leashing of real flying robots. In: Proceedings of the IEEE International Conference on Robotics and Automation, pp. 15-20 (2010)

5. Hauert, S., Winkler, L., Zufferey, J.-C., Floreano, D.: Ant-based swarming with positionless micro air vehicles for communication relay. Swarm Intelligence 2(2-4), 167-188 (2008)

6. Hauert, S., Zufferey, J.C., Floreano, D.: Evolved swarming without positioning information: an application in aerial communication relay. Autonomous Robots 26(1), 21-32 (2009)

7. Leven, S., Zufferey, J.C., Floreano, D.: A minimalist control strategy for small UAVs. In: Proceedings of the IEEE/RSJ International Conference on Intelligent Robots and Systems, pp. 2873-2878. IEEE Press, Piscataway (2009)

8. Li, Q., Jiang, Z.P.: Formation tracking control of unicycle teams with collision avoidance. In: Conference on Decision and Control, pp. 496-501. IEEE Press, Piscataway (2008) 
9. Mirollo, R.E., Strogatz, S.H.: Synchronization of pulse-coupled biological oscillators. SIAM Journal on Applied Mathematics 50, 1645-1662 (1990)

10. Moshtagh, N., Jadbabaie, A., Daniilidis, K.: Vision-based distributed coordination and flocking of multi-agent systems. In: Proceedings of Robotics: Science and Systems (2005)

11. Strogatz, S.H.: Sync: The Emerging Science of Spontaneous Order. Hyperion Press (2003)

12. Strogatz, S.H., Stewart, I.: Coupled oscillators and biological synchronization. Scientific American 269(6), 102-109 (1993)

13. Zufferey, J.C., Beyeler, A., Floreano, D.: Autonomous flight at low altitude with visionbased collision avoidance and GPS-based path following. In: Proceedings of the IEEE International Conference on Robotics and Automation. IEEE Press, Piscataway (2010)

14. Zufferey, J.C., Hauert, S., Stirling, T., Leven, S., Roberts, J., Floreano, D.: Handbook of Collective Robotics, chap. Aerial collective systems. Pan Stanford Publishing (in press) 